\title{
NANOCOMPÓSITOS DE MATRIZ POLIMÉRICA: ESTRATÉGIAS DE SÍNTESE DE MATERIAIS HÍBRIDOS
}

\author{
Ana Catarina C. Esteves, Ana Barros-Timmons e Tito Trindade* \\ Departamento de Química, CICECO, Universidade de Aveiro, 3810-193 Aveiro- Portugal
}

Recebido em 24/10/03; aceito em 5/2/04; publicado na web em 17/6/04

\begin{abstract}
POLYMER BASED NANOCOMPOSITES: SYNTHETIC STRATEGIES FOR HYBRID MATERIALS. Associating the well known advantages of hybrid materials to the wide potential of nanomaterials, the new and featuring class of polymer nanocomposites turned into one of the most intensively researched areas. This review highlights recent developments in the field of the synthesis of polymer based nanocomposites. Important issues related to the surface modification of fillers, in order to promote the compatibility between the inorganic/organic components, are also reported. The enhancement of the physical properties and the potential applications of polymer nanocomposites are considered in typical examples, given for each synthetic method described.
\end{abstract}

Keywords: nanocomposites; polymers; chemical synthesis

\section{INTRODUÇÃO}

Encontramos na mitologia grega um dos registos mais antigos da existência de híbridos como seres prodigiosos e fantásticos que povoaram a imaginação dos humanos. O grifo, por exemplo, aliava a força de um corpo de leão à perspicácia e capacidade de voar conferida pela cabeça e asas de uma águia, resultando num temeroso guardião do templo dos Deuses (Figura 1) ${ }^{1}$. Na própria natureza existem materiais híbridos admiráveis, sintetizados através de processos químicos em meio aquoso, sob condições de pressão e temperatura ambientes, com mecanismos de "automontagem" envolvendo interacções electroestáticas, ligações de pontes de hidrogénio ou forças de van der Waals ${ }^{2}$. Alguns dos nanocompósitos que ocorrem na natureza apresentam uma arquitectura molecular e sinergia perfeitas entre os seus componentes. Alguns exemplos típicos são o nacre (revestimento das pérolas) que consiste em lamelas nanométricas de aragonite $\left(\mathrm{CaCO}_{3}\right)$ dispersas numa mistura de proteínas e polissacarídeos, os ossos, os dentes e as conchas de alguns moluscos, que apresentam propriedades mecânicas extraordinárias ${ }^{2}$. Talvez seguindo esta bioinspiração os investigadores começaram por estudar uma forma de reproduzir estas propriedades, tentando aproximar-se do nível de

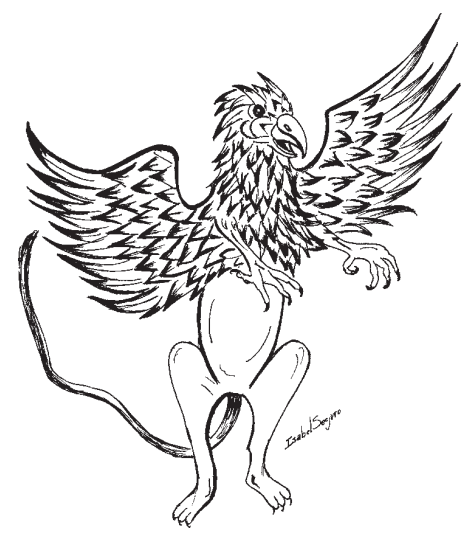

Figura 1. Grifo: ser híbrido da mitologia grega

\footnotetext{
*e-mail: ttrindade@dq.ua.pt
}

controlo da estrutura e propriedades dos compósitos naturais, numa tentativa de obter materiais avançados. A bio-mineralização é de facto um campo de investigação actual em grande desenvolvimento e com forte repercussão no campo dos materiais híbridos ${ }^{3,4}$. A tendência actual na área dos nanocompósitos é a preparação de materiais em que a interacção entre os componentes ocorre à escala nanométrica ou molecular. Os nanocompósitos obtidos deste modo, apresentam propriedades distintas dos compósitos tradicionais, podendo ser estabelecidas no processo de síntese ${ }^{5}$. Tem sido descrito na literatura um grande número de aplicações para os nanocompósitos, tais como em catálise ${ }^{6}$, optoelectrónica ${ }^{7}$, dispositivos magnéticos ${ }^{8}$, tintas e revestimentos $^{9}$ e como materiais retardadores de chama ${ }^{10}$.

Os nanocompósitos são materiais híbridos em que pelo menos um dos componentes tem dimensões nanométricas. Tal como acontece nos compósitos tradicionais, um dos componentes serve de matriz, na qual as partículas do segundo material se encontram dispersas. Os componentes de um nanocompósito podem ser de natureza inorgânica/inorgânica, inorgânica/orgânica ou ainda orgânica/orgânica. Esta revisão bibliográfica refere-se a nanocompósitos de matriz polimérica de interface inorgânica/orgânica. A incorporação de cargas inorgânicas em polímeros origina materiais com maior resistência mecânica ${ }^{11}$, maior estabilidade térmica ${ }^{12}$ ou com propriedades ópticas ${ }^{13}$, magnéticas ${ }^{14}$ ou eléctricas ${ }^{15}$ superiores. As cargas tendo dimensões nanométricas $(1-500 \mathrm{~nm})$ apresentam uma área de superfície elevada, promovendo melhor dispersão na matriz polimérica e por isso uma melhoria das propriedades físicas do compósito que dependem da homogeneidade do material. A utilização de nanocristais como cargas permite ainda alcançar propriedades interessantes que têm sido atribuídas a nanopartículas tais como efeitos quânticos de dimensão ${ }^{16,17}$, propriedades de transporte ${ }^{18}$ e propriedades magnéticas únicas ${ }^{19}$. Adicionalmente, a preparação de nanocompósitos de matriz polimérica permite em muitos casos encontrar um compromisso entre um baixo custo, devido à utilização de menor quantidade de carga, e um elevado nível de desempenho, que pode resultar da sinergia entre os componentes. O número elevado de publicações nos últimos anos versando nanocompósitos, nomeadamente artigos de revisão, reflectem o desenvolvimento que se tem verificado ao nível da preparação e caracterização deste tipo de materiais ${ }^{20,21}$. O estudo dos mecanismos de formação de híbridos, bem como das interacções que ocorrem ao nível da interface inorgânica/orgânica têm sido menos explorados 22,23 . 


\section{CARGAS: NANOPARTÍCULAS INORGÂNICAS}

A aplicação de nanopartículas como cargas é interessante, pelo facto destas apresentarem propriedades distintas dos materiais macrocristalinos quimicamente análogos. Vários autores têm descrito alterações nas propriedades ópticas, eléctricas e/ou mecânicas de um material, à medida que se passa da forma macrocristalina para um material constituído por partículas de dimensões nanométricas ${ }^{24-26}$. As propriedades físicas de nanomateriais semicondutores, nos quais ocorrem efeitos quânticos de dimensão, têm sido especialmente estudadas ${ }^{27}$. A fotoluminescência de nanocompósitos, preparados pela inserção de nanopartículas de semicondutores em matrizes poliméricas, foi investigada ${ }^{13,28}$. Em outro caso, partículas compósitas constituídas por nanocristalites de CdS suportadas em sílica, foram inseridas em copolímeros de estireno. Estes nanocompósitos apresentaram alterações significativas na microestrutura da matriz polimérica e a dispersão destas cargas nos copolímeros promoveu as propriedades ópticas do material semicondutor ${ }^{29}$.

As nanopartículas têm uma área de superfície elevada pelo que quando dispersas em matrizes poliméricas promovem alterações nas propriedades da matriz, relacionadas com a interacção química específica entre as cargas e o polímero. Este tipo de interacções pode influenciar a dinâmica molecular do polímero resultando em alterações significativas nas suas propriedades físicas, nomeadamente no seu comportamento térmico e/ou mecânico. Bershtein et al. ${ }^{30}$ investigaram um material híbrido constituído por nanopartículas de $\mathrm{SiO}_{2}$ inseridas em poli(imida). Os autores concluíram que a formação de ligações químicas entre as nanopartículas e determinados grupos terminais da poli(imida), confina o espaço acessível às cadeias poliméricas. Segundo estes investigadores, o confinamento espacial das cadeias poliméricas influencia a dinâmica molecular do polímero, resultando em alterações da sua estabilidade térmica e temperatura de transição vítrea $\left(\mathrm{T}_{\mathrm{g}}\right)^{30}$. Têm também sido descritas alterações da cristalização de matrizes poliméricas semicristalinas em nanocompósitos $^{31}$. Por exemplo, em um material híbrido constituído por uma matriz de poli(tetrametileno tereftalamida) contendo fibras ou esferas de $\mathrm{SiO}_{2}$ com dimensões nanométricas, foi registada uma influência da morfologia das nanopartículas na cinética de cristalização da poliamida $^{32}$. Em nanocompósitos de $\mathrm{Bi}_{2} \mathrm{~S}_{3} / \mathrm{Nylon}(6,10)$ verificou-se que as nanofibras de $\mathrm{Bi}_{2} \mathrm{~S}_{3}$ actuam como sítios de nucleação, resultando em um número maior de esferulites no nanocompósito relativamente ao polímero puro (Figura 2$)^{33}$.

Conforme a aplicação pretendida, podem ser usados diversos tipos de cargas que diferem entre si, por exemplo, nas propriedades morfológicas ou em propriedades tais como a resistência térmica, ou reactividade química. Entre as cargas mais comuns em nanocompósitos de matriz polimérica, encontram-se os carbonatos, os sulfatos, os alumino-silicatos e os óxidos metálicos $\left(\mathrm{Al}_{2} \mathrm{O}_{3}, \mathrm{Fe}_{2} \mathrm{O}_{3} \mathrm{ZnO}\right)^{5}$. As argilas e os silicatos lamelares, como por exemplo a montmorilonite (MMT) e a hectorite, são outro tipo de cargas que têm sido muito investigadas ${ }^{34-36}$. As nanopartículas de semicondutores, tais como CdS, ZnS, ou CdSe , têm também sido muito usadas devido às suas propriedades ópticas ${ }^{26,27}$. Alguns dos exemplos mais citados na literatura são nanopartículas de $\mathrm{TiO}_{2}$ na preparação de compósitos com aplicação na indústria de revestimentos e tintas ${ }^{9}$, e nanopartículas de $\mathrm{SiO}_{2}$ que podem conferir maior resistência mecânica ou características retardadoras de chama aos nanocompósitos ${ }^{10,11}$.

\section{MODIFICAÇ̃̃O QUÍMICA DE SUPERFÍCIES}

A afinidade química reduzida entre as cargas inorgânicas (natureza hidrofílica) e o polímero (predominantemente hidrofóbico) é um aspecto importante a considerar na preparação de nanocompósitos. A
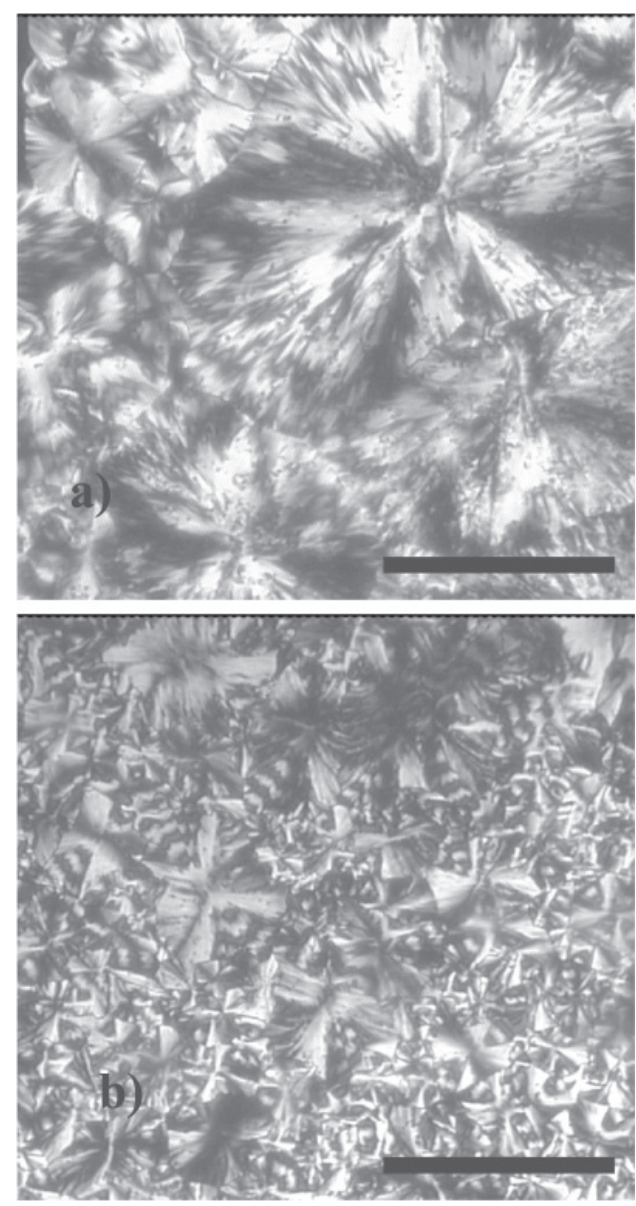

Figura 2. Imagens de microscopia óptica: a) Nylon 6,10; b) nano-Bi $\mathrm{S}_{3}$ / Nylon 6,10 (barra $=2 \mu \mathrm{m})(\text { cortesia da autora })^{33}$

compatibilidade das cargas com a matriz polimérica pode ser melhorada através da modificação química superficial das partículas dos componentes. Para o efeito, é normalmente utilizado um agente de derivatização, que promove a compatibilização química entres os componentes por intermédio de pontes de hidrogénio, interacções electroestáticas ou por ligações covalentes na interface inorgânica/orgânica. Na Figura 3 são apresentados alguns exemplos de alcoxi-silanos frequentemente utilizados na modificação de superfícies de sílica ${ }^{37-39}$.<smiles>C=CC(=O)OCCC[Si]OC</smiles>

MPTMS (3-metacriloxi-propil-trimetoxi-silano)<smiles>CO[SiH2]CCCOC1CO1</smiles>

GPTMS (3-glicidiloxi-propil-trimetoxi-silano)

$$
{ }_{2} \mathrm{HN} \sim \mathrm{Si}\left(\mathrm{OCH}_{3}\right)_{3}
$$

APMS (amino-propil-trimetoxi-silano)
$\mathrm{HS} \sim \mathrm{Si}\left(\mathrm{OCH}_{3}\right)_{3}$

SPTMS (sulfo-propil-trimetoxi-silano)

$\mathrm{NC} \sim \mathrm{Si}\left(\mathrm{OCH}_{3}\right)_{3}$

CPTMS (cianato-propil-trimetoxi-silano)

$$
\text { O } \mathrm{Si}\left(\mathrm{OCH}_{3}\right)_{3}
$$

PPTMS (fenil-propil-trimetoxi-silano)
Figura 3. Representação de alcoxi-silanos utilizados na modificação química de superfícies de sílica

Existem duas estratégias principais para estabelecer interacções químicas/físicas entre os componentes do nanocompósito. Enquanto que uma das estratégias passa pela passivação orgânica da superfície das partículas inorgânicas (Figura 4a); a outra consiste na inserção prévia de um monómero hidrofílico na cadeia polimérica (Figura 4b). Estas duas perspectivas serão seguidamente descritas, recor- 
rendo a exemplos de materiais híbridos contendo nanopartículas de $\mathrm{SiO}_{2}$, um dos tipos de cargas mais usadas na preparação de nanocompósitos de matriz polimérica. Os mesmos princípios de modificação de superfície podem ser adaptados para outros tipos de cargas e polímeros.

A Figura 4a esquematiza os procedimentos mais comuns na formação de um ambiente hidrofóbico à superfície de nanopartículas de $\mathrm{SiO}_{2}$. A estratégia mais simples (procedimento 1, Figura 4a) consiste em reacções de substituição na superfície da sílica. As partículas assim tratadas dispersam em solventes orgânicos e podem ser usadas posteriormente em métodos que envolvam polimerização em suspensão ou em emulsão. Foshiera et al $^{40}$ modificaram a superfície de partículas de sílica com amino-propil-metoxi-silano (APMS) (Figura 3), com o objectivo de acoplar moléculas orgânicas na superfície da sílica, tais como a anilina, a benzilamina e a $p$-anisidina. $\mathrm{O}$ material resultante consiste num suporte de sílica gel com grupos aromáticos imobilizados à superfície, que é utilizado como fase estacionária em cromatografia líquida. O APMS tem sido também usado como agente intermediário no revestimento com sílica de colóides de ouro, prata e cobalto ${ }^{41,42}$.

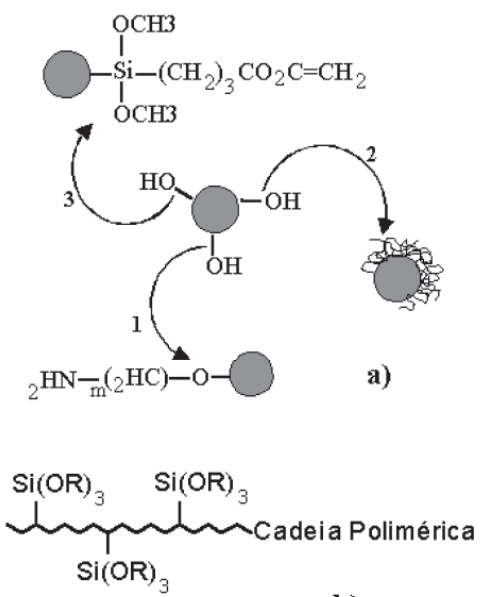

b)

Figura 4. a) Procedimentos de modificação química de superfícies inorgânicas: 1-esterificação à superfície; 2-adsorção de polímeros; 3ligação de moléculas orgânicas à superfície; b) Esquema de uma cadeia polimérica com grupos alcóxido pendentes

Outra forma de tornar a superfície de sólidos inorgânicos hidrofóbica é pela interacção de polímeros ou surfactantes orgânicos com a superfície das partículas inorgânicas (procedimento 2, Figura 4a). Neste caso o surfactante actua como estabilizante das partículas coloidais inorgânicas e das partículas poliméricas ${ }^{43}$. Utilizando este método, as cargas dispersam mais facilmente nos monómeros ou em solventes orgânicos. Contudo, a adição do estabilizante pode ter consequências indesejáveis na reologia e estabilidade da dispersão ${ }^{43}$. Por um lado o polímero pode evitar a agregação das partículas inorgânicas, mas por outro pode promover a sua floculação estabelecendo pontes entre as partículas. A quantidade de polímero adsorvida depende de vários parâmetros tais como o peso molecular, o tipo de unidades estruturais e o tipo do polímero (aleatório ou de blocos; linear ou ramificado; etc). Estes parâmetros são determinantes na interacção entre os segmentos do polímero e o sólido inorgânico. A composição da superfície da partícula inorgânica e as características do meio dispersante são também factores importantes a ter em conta. $\mathrm{O}$ pH da dispersão, por exemplo, determina na maior parte dos casos a carga da superfície inorgânica e, consequentemente, a natureza das interacções entre as duas fases ${ }^{44}$.
Finalmente, a outra estratégia de derivatização da superfície inorgânica, consiste na ligação de moléculas orgânicas na superfície do sólido inorgânico por intermédio de ligações covalentes (procedimento 3, Figura 4a). No caso de óxidos metálicos ricos em grupos hidroxílicos superficiais, estes reagem com moléculas orgânicas substituídas, do tipo RM'X $\mathrm{X}_{3}$, em que M' é um metal, X são grupos hidrolisáveis (aminas, halogéneos, ou alcóxido), e R é um grupo orgânico não hidrolisável (por ex: cadeias lineares longas), estabelecendose ligações do tipo M-O-M'. Os reagentes mais comuns neste procedimento são os cloro-silanos, os alcoxi-silanos e os organotitanatos. A reactividade de superfícies inorgânicas depende das condições de síntese, e por vezes o processo acima descrito requer um tratamento prévio da superfície. O número de grupos hidroxílicos superficiais é influenciado pela história térmica e por factores como o $\mathrm{pH}$ ou força iónica ${ }^{44}$. O controlo das espécies iónicas presentes na solução, pode ser também determinante no processo de derivatização da superfície, e da compatibilização das cargas com matrizes orgânicas ${ }^{44}$.

A outra estratégia referida para promover a compatibilidade entre as partículas inorgânicas e o polímero, consiste na inserção directa de unidades com carácter hidrofílico na cadeia polimérica da matriz (copolimerização) (Figura 4b). Outra alternativa é iniciar o processo de polimerização a partir de monómeros que possuam à priori grupos hidrofílicos/hidrofóbicos, tais como o 3-metacriloxi-propiltrimetoxi-silano (MPTMS) ou o 3-glicidiloxipropil-trimetoxi-silano (GPTMS $^{45}$ (Figura 3). Neste caso, podem obter-se materiais híbridos homogéneos, mas as condições de preparação têm de ser muito bem controladas. É necessário controlar a cinética das reacções de hidrólise e de condensação dos precursores inorgânicos; a cinética de polimerização da fase orgânica e a termodinâmica de separação das duas fases. As duas primeiras devem ocorrer rapidamente e em simultâneo, para minimizar efeitos de segregação dos componentes. $\mathrm{O}$ ajuste rigoroso das condições de hidrólise, ou o estabelecimento de ligações por pontes de hidrogénio podem ser usados para evitar a separação de fases. Chang et $a l .{ }^{46}$ prepararam um material híbrido de $\mathrm{SiO}_{2}$ /polivinilimidazole (PVI) pelo método sol-gel, inserindo previamente um monómero funcional com um grupo silano, o tetrametoxisilano (TMOS), na cadeia polimérica. O compósito preparado por estes investigadores apresentava um elevado grau de homogeneidade devido ao estabelecimento de ligações químicas entre o copolímero e a rede tridimensional da sílica.

\section{MÉTODOS DE SÍNTESE DE NANOCOMPÓSITOS DE MATRIZ POLIMÉRICA}

A preparação de nanocompósitos de matriz polimérica é uma área recente pelo que não existe ainda uma classificação inequívoca para os diferentes materiais híbridos e respectivos métodos de síntese. Uma das classificações que tem sido aceite baseia-se no tipo de ligações químicas que se estabelecem na interface inorgânica/orgânica $^{20}$. Segundo esta classificação distingue-se a Classe I para os híbridos que possuem ligações fracas entre os componentes (ligações de van der Waals, pontes de hidrogénio ou interacções electrostáticas); Classe II para os que apresentam ligações fortes entre a fase inorgânica/orgânica (ligações covalentes ou ionoméricas) ${ }^{20,47}$. A fronteira entre as duas classes é no entanto ténue, ocorrendo materiais que apresentam características comuns às duas categorias.

Nesta revisão bibliográfica a preparação de nanocompósitos de matriz polimérica é abordada segundo três estratégias principais (Figura 5): I) a mistura simples dos componentes;

II) a síntese das nanopartículas in situ e

III) a polimerização da matriz in situ.

Por vezes é usado mais do que um método na preparação do mesmo nanocompósito. A intercalação, por exemplo, consiste na inserção 


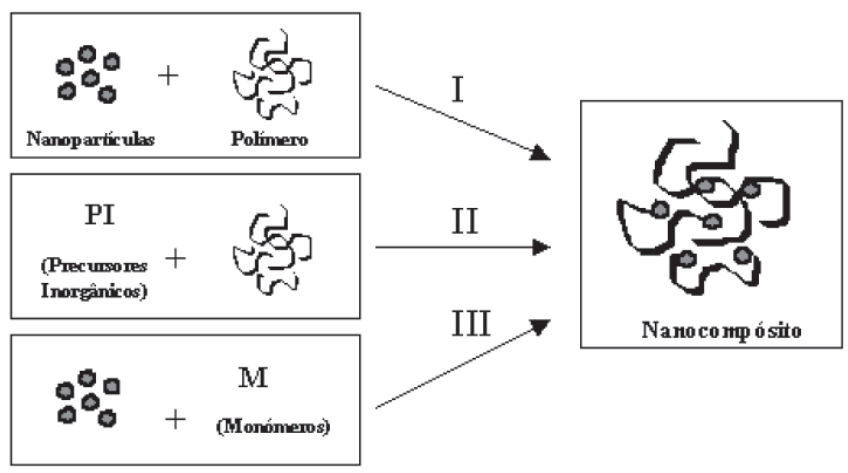

Figura 5. Estratégias de síntese de nanocompósitos: I) mistura simples dos componentes; II) preparação de nanopartículas in situ $e$ III) polimerização da matriz polimérica in situ

de um dos componentes na estrutura do outro e pode ser aplicada de diferentes formas. Na inserção do polímero em espaços vazios da estrutura do sólido inorgânico, considera-se a mistura simples (estratégia I); se os monómeros forem intercalados e posteriormente polimerizados considera-se a polimerização in situ (estratégia III).

Na síntese de nanocompósitos busca-se uma distribuição uniforme das cargas na matriz polimérica e uma boa adesão na interface dos dois componentes. Apesar do método de mistura simples fornecer bons resultados e ser uma forma expedita e económica de preparar materiais híbridos, a tendência é cada vez maior no sentido de preparar nanocompósitos com uma composição e microestrutura controlada. Os processos que envolvem a polimerização in situ, ou alternativamente, a síntese das cargas in situ, permitem um controlo à escala molecular sobre estes aspectos, tendo por isso vindo a ganhar um papel de destaque nesta área.

\section{Mistura de componentes}

O método da mistura simples dos componentes tem sido até agora o mais usado na preparação de compósitos ao nível industrial, principalmente em compósitos em que as cargas são estruturas lamelares ou em camadas, as quais apresentam maior flexibilidade quando comparadas com estruturas tridimensionais. As pequenas lamelas de compostos inorgânicos com dimensões tipicamente nanométricas são geralmente hidrofílicas, e antes de serem dispersas na matriz polimérica, têm de ser quimicamente modificadas para que se tornem compatíveis com os polímeros. Um dos sólidos lamelares mais usados tem sido a montmorilonite (MMT), uma argila que na sua estrutura apresenta tetraedros de silicatos que partilham um dos vértices com folhas octaédricas de $\mathrm{Al}(\mathrm{OH})_{3}$ e $\mathrm{Mg}(\mathrm{OH})_{2}$. O empacotamento das camadas é efectuado através de forças de van der Waals originando um espaço vazio, muitas vezes denominado galeria interlamelar. Tien et al..$^{48}$ prepararam um nanocompósito a partir da intercalação de um poli(uretano) (PU) entre camadas de MMT. O silicato foi previamente modificado com benzidina, disperso em dimetilformamida (DMF) e posteriormente misturado com uma solução do PU em DMF. A mistura ficou em agitação durante $3 \mathrm{~h}$ à temperatura ambiente e finalmente foi obtido o nanocompósito MMT/PU.

Têm sido preparados vários compósitos através deste processo de mistura simples, principalmente com matrizes poliméricas do tipo poli(estireno), poli(vinilideno), poli(propileno), poli(imida), poli(éster) ou poli(amidas) do tipo nylon 6 e nylon $6,6^{5}$. Suh et al. ${ }^{49}$ prepararam um nanocompósito do tipo MMT/poliéster insaturado (PE) por mistura dos componentes. Estes autores referem que as características do híbrido dependem do processo de síntese, das reacções químicas e das interacções físicas envolvidas no processo.
Foram usados dois métodos distintos para investigar as propriedades e o mecanismo de formação deste nanocompósito ${ }^{49}$. Em ambos os casos a MMT foi previamente tratada com brometo de dodecilamónio para se obter uma superfície hidrofóbica. No primeiro método o poliéster foi misturado com estireno, e mantido em agitação durante 180 min a $60{ }^{\circ} \mathrm{C}$; seguidamente foi adicionada a MMT. No segundo processo, os autores efectuaram uma mistura sequencial, em que no primeiro passo o PE foi pré-intercalado entre as lamelas do silicato e só depois adicionaram o estireno, variando os tempos de mistura entre 15 e $180 \mathrm{~min}$, a $60^{\circ} \mathrm{C}$. Nos dois processos o material resultante foi aquecido a $120^{\circ} \mathrm{C}$ durante $4 \mathrm{~h}$, para promover as reticulações entre o poliéster e o estireno. A estrutura dos nanocompósitos foi estudada por difracção de raios-X e TEM, e o mecanismo de formação foi investigado por várias técnicas de análise térmica, mecânica e reológica. Os autores concluíram que no caso da mistura sequencial, o estireno se difunde mais facilmente para a galeria da MMT, onde já foi previamente intercalado o $\mathrm{PE}^{49}$. $\mathrm{O}$ estireno, que actua como um agente promotor de reticulações está, neste caso, homogeneamente disperso no sistema e assim, a densidade de reticulações aumenta em grande extensão, relativamente ao nanocompósito obtido por mistura simultânea. Em conformidade com esta observação, os autores registaram que a $\mathrm{T}_{\mathrm{g}}$ do nanocompósito MMT/ PE preparado pela mistura sequencial é muito superior relativamente aos nanocompósitos preparados pelo primeiro processo.

No caso de cargas com estrutura lamelar, a preparação dos nanocompósitos de matriz polimérica através da mistura simples dos componentes tem dado bons resultados. No entanto, com cargas com outras estruturas este método pode originar materiais pouco homogéneos. No que concerne à mistura dos componentes destacam-se em particular os três métodos de preparação seguintes.

\section{Intercalação por fusão}

Este método é ecologicamente favorável uma vez que não é necessário utilizar solventes orgânicos voláteis. O procedimento consiste no aquecimento de uma mistura do polímero e das cargas inorgânicas, a uma temperatura superior ao ponto de fusão para polímeros semicristalinos, ou acima da $\mathrm{T}_{\mathrm{g}}$ para os polímeros amorfos. Rong et al. ${ }^{50}$ prepararam um nanocompósito de $\mathrm{SiO}_{2} /$ poli(propileno), partindo de nanopartículas de $\mathrm{SiO}_{2}$ previamente tratadas com poliestireno. As cargas modificadas foram misturadas com o polipropileno numa extrusora. Os investigadores obtiveram assim um nanocompósito com propriedades mecânicas melhoradas ${ }^{50}$.

No que diz respeito à dispersão de cargas lamelares em um polímero, determinados segmentos poliméricos adquirem mobilidade suficiente e podem difundir-se para o interior das lamelas. O grupo de Giannelis ${ }^{51}$ foi pioneiro nesta estratégia ao preparar nanocompósitos constituídos por silicatos lamelares e poli(óxido de etileno) (PEO) ou poli(estireno) (PS). Os autores registaram alterações significativas ao nível do comportamento térmico destes híbridos (nomeadamente na $\mathrm{T}_{\mathrm{g}}$ do polímero), que tornam estes materiais promissores para aplicações em que a adesão, a lubrificação ou a fricção, são de extrema importância, tais como na preparação de lubrificantes, adesivos ou filmes ultra-finos ${ }^{49}$. Estes nanocompósitos podem ser processados por métodos utilizados frequentemente em tecnologia de polímeros, tal como a extrusão.

\section{Exfoliação/adsorção}

Alguns materiais lamelares como as argilas e certos dicalcogenetos de metais de transição, podem ser parcial ou totalmente delaminados. Normalmente, a delaminação é promovida pela introdução de espécies químicas entre as camadas inorgânicas, como por exemplo aminas com cadeias alquílicas longas ou sais de amónio. A exfoliação/adsorção é um método viável para a preparação de 
nanocompósitos se o polímero em questão for solúvel num determinado solvente, no qual o componente inorgânico possa ser delaminado.

Certos materiais inorgânicos tais como o $\mathrm{MoO}_{3}$ e o $\mathrm{MoS}_{2}$ podem ser pré-intercalados com iões $\mathrm{Li}^{+}$, através de reacções químicas/ electroquímicas para aumentar a delaminação. O processo é normalmente completado por agitação mecânica e/ou aplicação de ultrasons. Quando é adicionada uma solução do polímero, as folhas delaminadas organizam-se espontaneamente para formar nanocompósitos ordenados, com o polímero inserido entre as lamelas (Figura 6$)^{5,52,53}$. Em alguns casos o polímero intercalado pode ser posteriormente removido de uma forma quantitativa, tal como descrito por Oriakhi et al. ${ }^{53}$ para nanocompósitos do tipo $\mathrm{MoS}_{2} / \mathrm{PEO}$ e $\mathrm{MoO}_{3} / \mathrm{PEO}$. Os nanocompósitos deste tipo podem ser usados por exemplo como electrólitos sólidos em baterias de Li recarregáveis, devido à sua condutividade iónica, e à estabilidade interfacial e mecânica $^{54}$. Uma grande parte dos polímeros existentes são no entanto pouco solúveis nos solventes orgânicos comuns, o que limita a sua utilização para preparar nanocompósitos através deste método.

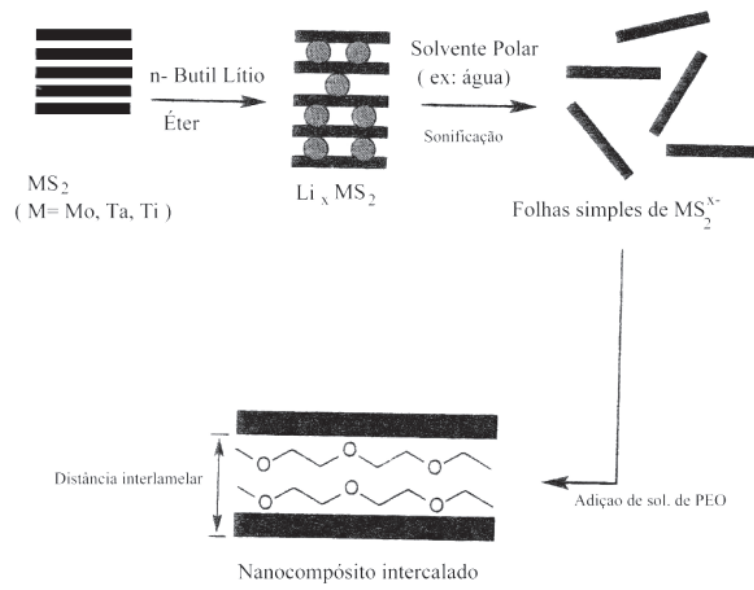

Figura 6. Preparação de nanocompósitos lamelares contendo poli(óxido de etileno) através do processo de exfoliação/adsorção. Reproduzida da ref. 5, com permissão do J. Chem. Educ.

\section{Utilização de materiais micro e mesoporosos}

Os materiais micro e mesoporosos possuem poros ou canais estáveis bem definidos e com dimensões que vão desde 2 a $500 \AA$ A Um dos métodos de preparar nanocompósitos com estes materiais é inserir directamente as moléculas do polímero nos poros ou canais dos sólidos inorgânicos. Tal como no caso anterior, esta técnica só é aplicável a polímeros solúveis ou que depois de fundidos, possam difundir facilmente para o interior dos poros da estrutura inorgânica. Com materiais poliméricos de elevado peso molecular, podem ainda surgir limitações adicionais devido ao tamanho das macromoléculas, às diferentes conformações ou à fraca mobilidade. Frish et $a l .{ }^{55}$ prepararam nanocompósitos através da mistura de dois tipos de materiais microporosos (zeólitos 3A e 13X) com poli(dimetilsioxano) (PDMS). Os autores registaram para ambos os compósitos uma melhoria das propriedades mecânicas do elastómero (PDMS), em particular para o nanocompósito contendo o zeólito com poros de maior dimensão (zeólito $13 \mathrm{X})^{55}$.

\section{Síntese de cargas in situ}

A síntese das cargas envolve métodos químicos de preparação controlada de sólidos inorgânicos. Os materiais preparados segundo esta estratégia apresentam, geralmente, ligações químicas entre os componentes, o que resulta em híbridos mais homogéneos e com maior consistência. Existem dois métodos principais para preparar nanocompósitos por síntese das cargas in situ, que serão descritos seguidamente.

\section{Métodos envolvendo reacções sol-gel}

$\mathrm{Na}$ última década o processo sol-gel tem sido muito usado na preparação de novos materiais híbridos, principalmente para aplicações em sistemas ópticos onde é requerida grande homogeneidade e transparência dos materiais ${ }^{56}$. No caso dos nanocompósitos de matriz polimérica, este método permite a formação das cargas na presença de polímeros com grupos funcionais que estabeleçam interacções com a fase inorgânica. As reacções sol-gel ocorrem a partir de precursores inorgânicos do tipo $\operatorname{ROM}\left(\mathrm{OR}^{\prime}\right)_{3}$ em que frequentemente $\mathrm{M}$ é $\mathrm{Si}$ ou Ti, e R pode ser igual a R' ou, alternativamente, ser um grupo orgânico não hidrolisável. As reacções que se pensa estarem envolvidas neste processo são a hidrólise (reacção 1), em meio ácido ou básico, na qual os grupos OR são substituídos por grupos silanol (Si-OH). Estes grupos silanol podem reagir entre si, (reacção 2) ou com outros grupos OR (reacção 3) através de reacções de condensação formando ligações siloxano, dando origem a uma rede tridimensional de sílica. À medida que a condensação vai ocorrendo o solvente fica retido no interior dos poros da estrutura, e obtém-se um gel que se torna progressivamente mais denso ${ }^{51}$.

$$
\begin{array}{ll}
\mathrm{Si}-\mathrm{OR}+\mathrm{H}_{2} \mathrm{O} & \stackrel{\mathrm{H}^{+} / \mathrm{OH}^{-}}{\longrightarrow} \mathrm{SiOH}+\mathrm{R}-\mathrm{OH} \\
\mathrm{Si}-\mathrm{OH}+\mathrm{HO}-\mathrm{Si} \stackrel{\mathrm{H}^{+} / \mathrm{OH}^{-}}{\longrightarrow} \mathrm{Si}-\mathrm{O}-\mathrm{Si}+\mathrm{H}_{2} \mathrm{O} \\
\mathrm{Si}-\mathrm{OH}+\mathrm{RO}-\mathrm{Si} \stackrel{\mathrm{H}^{+} / \mathrm{OH}^{-}}{\longrightarrow} \mathrm{Si}-\mathrm{O}-\mathrm{Si}+\mathrm{ROH}
\end{array}
$$

Na preparação de nanocompósitos utilizando o método sol-gel, a hidrólise dos grupos OR' dos derivados alcóxido ocorre na presença da matiz polimérica, seguida de reacções de condensação que levam à formação de um gel no qual o polímero fica retido ${ }^{56}$. Uma das principais vantagens deste método é que os nanocompósitos obtidos são de forma geral muito puros e homogéneos, e por outro lado, as temperaturas de reacção são moderadas (normalmente temperatura ambiente), evitando-se a degradação do polímero. A morfologia e tamanho das partículas são controlados durante o processo de síntese e as características finais do nanocompósito são influenciadas por vários parâmetros experimentais, tais como a concentração relativa dos reagentes ou o $\mathrm{pH}$. O compósito resultante pode ser disperso em solventes orgânicos e processado, por exemplo, para preparar filmes poliméricos $^{47}$.

Existem disponíveis no mercado diversos precursores inorgânicos de $\mathrm{SiO}_{2}$, como por exemplo o tetraetoxi-silano (TEOS- $\mathrm{Si}\left(\mathrm{OC}_{2} \mathrm{H}_{5}\right)_{4}$ ), que por hidrólise controlada origina partículas inorgânicas de $\mathrm{SiO}_{2}$ de elevada pureza ${ }^{45}$. Os tetra-alcoxi-silanos são frequentemente usados pois tendem a formar uma rede tridimensional de sílica altamente ramificada o que resulta em nanocompósitos mais consistentes. Os tri-alcoxi-silanos do tipo $\mathrm{R}^{\prime} \mathrm{Si}\left(\mathrm{OC}_{2} \mathrm{H}_{5}\right)_{3}$ em regra, não formam uma rede tão densa, mas têm sido também utilizados na preparação de híbridos com matrizes poliméricas. Uma vez que o grupo R' não é hidrolizável, fica livre para reagir quimicamente e promover a compatibilidade das cargas com a matriz polimérica ${ }^{45}$.

No que diz respeito ao componente orgânico, têm sido usados vários polímeros, em particular os que apresentam capacidade de formar pontes de hidrogénio com os materiais inorgânicos, tais como elastómeros (PDMS), polímeros amorfos (PMMA, PVAc) e polímeros semicristalinos (Nafion, Nylon). Os compósitos resultan- 
tes podem assim apresentar-se duros ou flexíveis, quebradiços ou resistentes, dependendo da estrutura química dos componentes orgânicos, da composição relativa do compósito, e das interacções entre as fases inorgânica/orgânica ${ }^{47}$.

Chen et al. ${ }^{57}$ prepararam um novo nanocompósito de $\mathrm{SiO}_{2} /$ poli(imida) (PI), em que o polímero e as cargas inorgânicas se encontram ligados quimicamente. O alcoxi-silano APTMS, foi previamente inserido na cadeia polimérica, o que permitiu o estabelecimento de interacções entre a matriz e a estrutura de rede da sílica. O gel resultante foi colocado em substratos de vidro e após tratamento térmico foram obtidos filmes do nanocompósito ${ }^{57}$. Estes filmes nanocompósitos não evidenciaram qualquer alteração na temperatura de decomposição e a sua energia de superfície aumenta com a quantidade de sílica no compósito, o que se traduziu numa melhor adesão dos filmes ao substrato. Os autores concluíram assim, que a introdução de ligações químicas entre os componentes, aumentou a sua compatibilidade e melhorou as propriedades mecânicas dos filmes do nanocompósito $\mathrm{SiO}_{2} / \mathrm{PI}$. Partindo de um outro precursor inorgânico (TMOS), Chang et al. ${ }^{46}$ prepararam também um híbrido de $\mathrm{SiO}_{2} /$ poli(vinil imidazole) (PVI) homogéneo e que apresenta igualmente ligações químicas entre o copolímero e a rede tridimensional da sílica, promovendo uma melhoria das propriedades térmicas do nanocompósito. O processo sol-gel é um método versátil para a preparação de materiais híbridos uma vez que pode ser aplicado a uma grande variedade de precursores, ou até a misturas que originam sólidos inorgânicos compósitos. Um exemplo interessante é o caso apresentado por Shao et al. ${ }^{58}$, em que os autores prepararam nanocompósitos do tipo $\left(\mathrm{TiO}_{2} / \mathrm{SiO}_{2}\right) / \mathrm{Nafion}$ e $\left(\mathrm{SiO}_{2} / \mathrm{Al}_{2} \mathrm{O}_{3}\right) / \mathrm{Nafion}$. As cargas foram preparadas in situ, a partir de uma mistura de TEOS e de alcóxidos do tipo $\mathrm{Ti}(\mathrm{OR})_{4}$ e $\mathrm{Al}(\mathrm{OR})_{3}$ na presença do material polimérico ${ }^{58}$.

\section{Síntese na presença de materiais estruturantes}

Um outro método de preparação de nanocompósitos de matriz polimérica envolve a síntese das cargas inorgânicas na presença de materiais estruturantes. Neste método procede-se à cristalização de estruturas ordenadas em camada, a partir de uma solução aquosa homogénea dos precursores inorgânicos que contém o polímero, e através de um mecanismo de "auto-montagem". Através deste mecanismo o material polimérico fica retido no interior das camadas durante a formação dos cristais inorgânicos. Este método foi descrito por Oriakhi et al. ${ }^{5}$ para a preparação de nanocompósitos com hidróxidos de metais de camada dupla (estruturas lamelares). Messersmith et al..$^{59}$ prepararam também um nanocompósito constituído por nanocamadas lamelares de hidróxido de cálcio e alumínio contendo o polímero poli(vinil álcool) (PVA) intercalado. O sólido lamelar de camada dupla foi preparado a partir de uma solução contendo o PVA e precursores dos respectivos hidróxidos. No caso da aplicação deste método a cargas inorgânicas lamelares é preferível a utilização de polímeros neutros, de forma a minimizar repulsões electroestáticas entre os componentes.

\section{Síntese da matriz polimérica in situ}

As propriedades de um nanocompósito são em grande medida determinadas pela matriz que, neste caso particular, é um material polimérico. Os polímeros constituem um excelente suporte para as nanopartículas, protegendo-as da degradação física/química e facilitando a sua manipulação/ processamento. A síntese controlada do polímero na presença de nanopartículas inorgânicas, possibilita um controlo rigoroso sobre as propriedades físicas/químicas da matriz. Permite ainda obter uma boa dispersão das cargas, originando nanocompósitos homogéneos de fácil processamento e baixo custo de produção.
Têm sido descritas na literatura variações no comportamento térmico e mecânico de vários tipos de polímeros, resultantes da inserção de partículas com dimensões nanométricas em matrizes poliméricas ${ }^{12,30}$. Entre os polímeros mais usados na preparação de nanocompósitos destacam-se as poli(olefinas), os poli(ésteres) insaturados, as resinas epóxi, as poli(amidas) e poli(imidas), nomeadamente para o fabrico de materiais com características retardadoras de chama ${ }^{60,61}$. Os polímeros condutores, tais como a poli(anilina) ou o poli(pirrole), têm também atraído a atenção de investigadores na preparação de nanocompósitos com vista a aplicações em dispositivos electrónicos e magnéticos ${ }^{62,63}$. Outras matrizes muito utilizadas são os polímeros vinílicos, copolímeros de estireno, e o próprio PS, em particular nos processos de encapsulamento de partículas inorgânicas para aplicações em tintas e revestimentos ${ }^{64,65}$. Esta estratégia de preparação de nanocompósitos é aqui apresentada tendo em conta as técnicas de síntese de polímeros mais utilizadas: a polimerização em dispersão, a polimerização em emulsão e a polimerização em massa. O desenvolvimento recente no que concerne a novos métodos de polimerização radicalar, correntemente denominados por métodos de polimerização controlada ou "viva", vieram facultar novas metodologias para preparar materiais poliméricos de forma controlada. No entanto, estes métodos apresentam ainda algumas limitações no que diz respeito à especificidade e custo dos agentes mediadores da polimerização ${ }^{66}$.

\section{Polimerização em dispersão}

Na polimerização em dispersão, o iniciador e os monómeros são solúveis na fase contínua. A reacção de polimerização ocorre na presença de um estabilizante ${ }^{67}$. Na preparação de nanocompósitos utilizando esta técnica, efectua-se a dispersão das cargas inorgânicas no meio reaccional, ou no próprio monómero, e só depois se dá início à polimerização da matriz. Sondi et al. ${ }^{65}$ prepararam um nanocompósito de $\mathrm{SiO}_{2} /$ poli(acrilato de butilo) por polimerização in situ em dispersão. As nanopartículas de sílica foram previamente derivatizadas com o alcoxi-silano MPTMS (Figura 3), e dispersas em 2-propanol, onde foi iniciada a polimerização da matriz na presença do iniciador azobisisobutironitrilo (AIBN). O material resultante apresentava uma fotoresistência superior ao polímero de base e características adequadas para a sua utilização em filmes ${ }^{65}$.

Bourgeat-Lami et al. ${ }^{68}$ descreveram o encapsulamento de partículas de sílica derivatizadas com MPTMS, com poli(estireno), através de polimerização radicalar in situ em dispersão. A fase contínua consistiu numa mistura de água/etanol (5:95), o estabilizante usado foi a poli(n-vinil pirrolidona) (PVP) e o AIBN o iniciador. Os autores observaram a formação de partículas compósitas com morfologia do tipo núcleo de $\mathrm{SiO}_{2}$-coroa de $\mathrm{PS}$, e ainda que o número de nanopartículas de $\mathrm{SiO}_{2}$ no interior de cada partícula de nanocompósito, varia com a concentração destas na dispersão inicial ${ }^{68}$.

Barthet et al. ${ }^{68}$ prepararam também por polimerização in situ em dispersão uma série de nanocompósitos de $\mathrm{SiO}_{2}$ com várias matrizes poliméricas, tais como a poli(4-vinilpiridina) ou um copolímero de estireno e 4-vinilpiridina. O meio reaccional consistiu igualmente numa mistura de água/etanol mas, contrariamente ao caso anterior, as nanopartículas não foram sujeitas a nenhum tratamento prévio de derivatização, nem foram utilizados quaisquer estabilizantes. Neste caso, o processo de estabilização coloidal do sistema resultou do comportamento ácido-base entre as partículas de sílica e monómeros de elevada basicidade ${ }^{69}$.

\section{Polimerização em emulsão}

A utilização da técnica de polimerização em emulsão para preparar materiais híbridos, requer um controlo rigoroso de vários parâmetros, que podem ser determinantes na formação dos 
nanocompósitos. O meio contínuo neste tipo de polimerização é geralmente a água. $\mathrm{O}$ processo envolve um iniciador solúvel na fase contínua, um surfactante e monómeros insolúveis ou pouco solúveis em água, que se encontram sob a forma de gotas estabilizadas pelo surfactante $^{60}$. A reacção de polimerização ocorre preferencialmente nas partículas poliméricas em crescimento e no interior das micelas do surfactante, mas pode também ocorrer parcialmente na fase contínua. As duas últimas possibilidades originam partículas de polímero livre.

A principal vantagem deste método é o facto de permitir algum controlo sobre a massa molecular e a distribuição de massas moleculares do polímero, através da variação de factores como a concentração do iniciador e/ou do tensioactivo. As partículas poliméricas resultantes apresentam uma forma e tamanho específico (geralmente esféricas e com 0,05-5 $\mu \mathrm{m}$ de diâmetro) ${ }^{67}$.

Choi et al. ${ }^{70}$ prepararam nanocompósitos de poli(metacrilato de metilo) (PMMA) contendo cargas de MMT, através de um processo de polimerização in situ em emulsão. A MMT foi previamente dispersa em água e misturada com o monómero, numa solução aquosa contendo o tensioactivo. Foram intercalados na MMT dois tipos de surfactantes: um aniónico convencional (dodecilbezenosulfonato de sódio: DBS-Na) e um surfactante reactivo (ácido 2-acrilamido-2metil-1-propenosulfónico: AMPS). A polimerização foi realizada utilizando um iniciador solúvel na fase aquosa, à temperatura de $65^{\circ} \mathrm{C}$. Os autores constataram que em ambos os casos, as lamelas exfoliadas do silicato se apresentavam homogeneamente dispersas na matriz ${ }^{70}$. Registaram no entanto uma $\mathrm{T}_{\mathrm{g}}$ mais elevada para os nanocompósitos preparados com AMPS, facto este que justificam com a ocorrência de maior delaminação dos silicatos promovendo a interacção com o PMMA. No caso do material preparado com o DBS-Na, os autores registaram uma $\mathrm{T}_{\mathrm{g}}$ mais baixas para os nanocompósitos, o que atribuíram a um efeito plasticizante do surfactante intercalado na $\mathrm{MMT}^{70}$.

A polimerização em emulsão tem sido também muito usada em reacções de encapsulamento de partículas inorgânicas. Esta estratégia foi utilizada na preparação de um nanocompósito de $\mathrm{SiO}_{2} / \mathrm{PS}$ utilizando um surfactante comercial não iónico (Triton X 405) e partindo de nanopartículas de $\mathrm{SiO}_{2}$ previamente dispersas na fase aquosa. No nanocompósito final foram identificadas partículas de $\mathrm{SiO}_{2}$ encapsuladas com morfologia do tipo "núcleo-coroa" (Figura 7) 7 "1. $^{2}$. Este trabalho mostrou ainda que as condições do meio reaccional ( $\mathrm{pH}$ e surfactante) e a derivatização da superfície do sólido inorgânico, podem ser determinantes na eficiência do encapsulamento, assim como na morfologia das partículas do nanocompósito ${ }^{71}$.

Bourgeat-Lami ${ }^{38}$ sugeriu um possível mecanismo para o processo de polimerização em emulsão a partir da superfície de óxidos inorgânicos, segundo três etapas principais (Figura 8):

I) o surfactante adsorve à superfície do sólido inorgânico, formando agregados micelares em volta deste;

II) o monómero é solubilizado nas micelas adsorvidas à superficie e

III)ocorre a reacção de polimerização a partir dos monómeros e dos oligoradicais presentes no interior das micelas adsorvidas, obtendo-se assim materiais inorgânicos encapsulados com polímero ${ }^{66}$.

Luna-Xavier et al. ${ }^{72}$ prepararam um nanocompósito de $\mathrm{SiO}_{2} /$ PMMA, recorrendo à polimerização em emulsão na presença de um iniciador catiónico 2,2'-isobutiroamidina (AIBA) e de um surfactante não iónico (NP30). Variando as condições do meio tais como o pH, a concentração do monómero ou a quantidade de iniciador, os autores investigaram a natureza das interacções químicas que controlam o mecanismo de formação do nanocompósito. Desse trabalho concluíram que valores elevados de $\mathrm{pH}$ parecem favorecer as interacções electrostáticas entre a superfície das nanopartículas de sílica e as cadeias poliméricas, em relação às interacções hidrofóbicas ${ }^{72}$. Por ou-
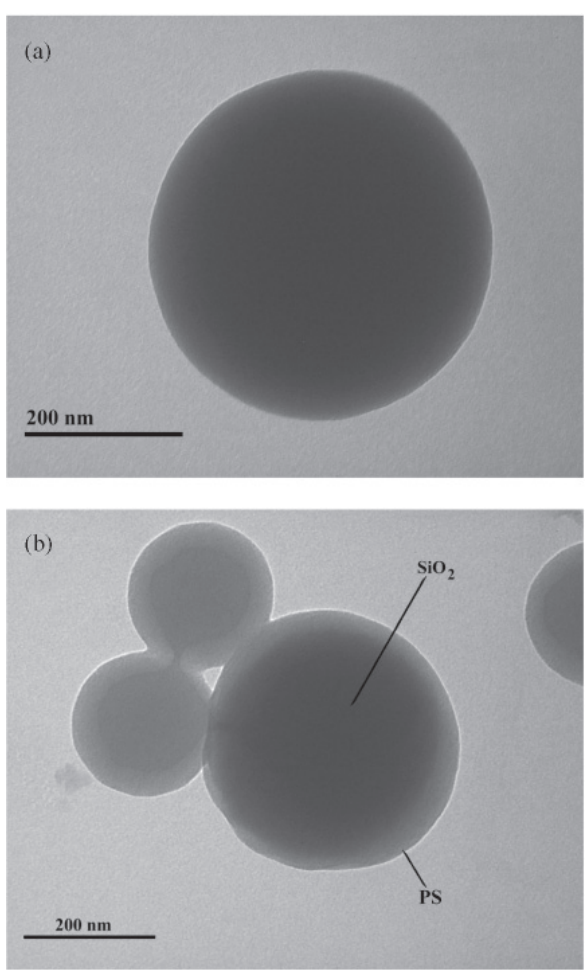

Figura 7. Imagens de microscopia electrónica de transmissão (TEM): a) nanopartícula de $\mathrm{SiO}_{2}$; b) partícula do nanocompósito $\mathrm{SiO}_{2} /$ poli(estireno) com morfologia "núcleo-coroa”, preparado por polimerização in situ em emulsão ${ }^{71}$

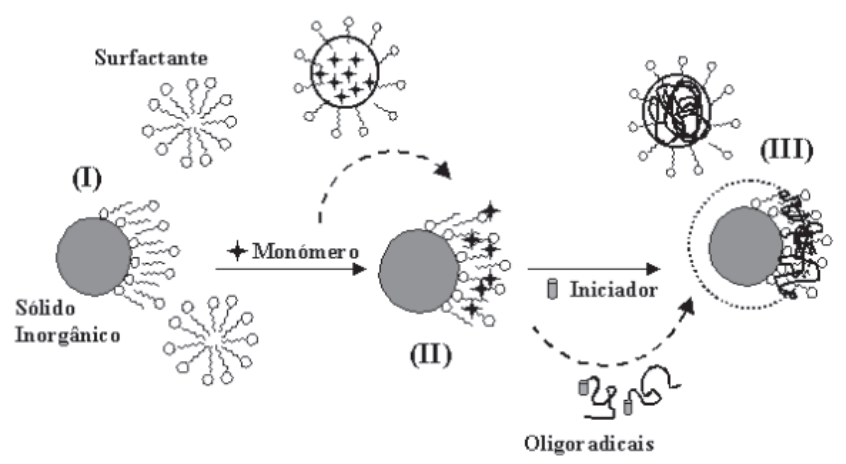

Figura 8. Encapsulamento de partículas inorgânicas através de um mecanismo de polimerização em emulsão: (I) formação da bicamada de surfactante na superfície das nanopartículas; (II) solubilização dos monómeros; (III) polimerização dos monómeros no interior das micelas

tro lado, concluíram que uma quantidade de monómero e de iniciador elevada promove a formação de partículas de polímero livre na fase contínua, diminuindo a eficiência do encapsulamento ${ }^{72}$.

\section{Polimerização em massa}

Na preparação de nanocompósitos de matriz polimérica por polimerização em massa, a reacção é relativamente rápida e ocorre na ausência de solventes. Este tipo de polimerização apenas envolve os monómeros, e pode ser induzida por um iniciador químico, por aplicação de uma fonte térmica ou por fontes radiativas, o que permite obter nanocompósitos com elevada pureza. Esta técnica implica no entanto um dispêndio maior de energia uma vez que a reacção 
tem lugar à temperatura de fusão dos monómeros que, tal como com o polímero, têm de ser miscíveis entre si. O facto de se obter um produto final com elevada viscosidade pode em alguns casos dificultar a dispersão das cargas inorgânicas, mas esta técnica é tecnologicamente um dos processos mais fáceis de implementar ao nível industrial, nomeadamente pela utilização de extrusoras ${ }^{67}$.

Avella et ll $^{73}$ prepararam materiais híbridos de PMMA contendo nanopartículas de $\mathrm{CaCO}_{3}$ derivatizadas à superfície, por polimerização em massa in situ. Os polímeros acrílicos são normalmente polimerizados em suspensão, mas neste caso a polimerização foi efectuada em massa, devido à incompatibilidade das nanopartículas com a água. O monómero metacrilato de metilo (MMA), e o iniciador, peróxido de dicumilo (DCPO), foram inseridos num reactor. Seguidamente foram adicionadas quantidades diferentes de $\mathrm{CaCO}_{3}$ (2 a $6 \mathrm{wt} \%$ ) previamente tratado com ácido esteárico para aumentar o seu carácter hidrofóbico, seguindo-se a polimerização a $90{ }^{\circ} \mathrm{C}$. O nanocompósito final apresentava-se homogéneo, e foi obtida uma boa dispersão das cargas na matriz, mesmo para os compósitos contendo quantidades mais elevadas de $\mathrm{CaCO}_{3}(6 \mathrm{wt} \%)^{73}$. A caracterização térmica destes nanocompósitos revelou um aumento da $\mathrm{T}_{\mathrm{g}} \mathrm{de}$ aproximadamente $30{ }^{\circ} \mathrm{C}$ em relação ao PMMA, devido à presença do sólido inorgânico. Em termos de propriedades mecânicas, Avella et al. ${ }^{73}$ verificaram um aumento do módulo de Young e da resistência ao desgaste nos materiais híbridos, em relação ao polímero simples, mesmo em nanocompósitos com menores quantidades de $\mathrm{CaCO}_{3}$. Segundo estes investigadores, estes dados estão relacionadas com o facto do sólido inorgânico suportar parte da carga aplicada.

Um outro exemplo interessante da utilização da polimerização em massa, na síntese de híbridos de matriz polimérica, é o trabalho descrito por Liu et al. ${ }^{36}$. Estes autores prepararam nanocompósitos a partir da polimerização in situ da 2-etil-piridina previamente intercalada no interior das camadas lamelares de MMT. O sólido inorgânico para além de funcionar como carga e matriz receptora dos monómeros, funciona também como iniciador, uma vez que a polimerização dos monómeros ocorre espontaneamente.

A estratégia de preparação de nanocompósitos de matriz polimérica através de polimerização in situ na presença das nanopartículas, requer um conhecimento prévio das condições e do mecanismo de polimerização, assim como a selecção criteriosa dos monómeros adequados. Porém uma vez estabelecidas as condições, permite preparar nanofilamentos, fibras ou até filmes finos. Se os monómeros possuírem o tamanho e a geometria apropriada podem ainda ser inseridos nos poros ou canais de uma estrutura micro ou mesoporosa por troca iónica ou inclusão directa, através de um gás ou de uma fase líquida. Este método tem sido na verdade referido na literatura como uma das estratégias mais promissoras, e a tendência parece apontar para a sua aplicação a uma gama cada vez mais variada, quer de cargas inorgânicas quer de matrizes poliméricas.

\section{CONCLUSÃO}

As estratégias mais frequentes na preparação de nanocompósitos de matriz polimérica foram aqui revistas. Após uma primeira fase centrada na síntese de novos nanocompósitos, perspectiva-se uma maior atenção ao estudo das interacções químicas que ocorrem na interface inorgânica/orgânica. O conhecimento da química-física de interfaces é crucial para o desenvolvimento de nanocompósitos com propriedades inovadoras. Ao longo do texto, subentende-se que o desenvolvimento de métodos preparativos de nanocompósitos para aplicações tecnológicas é indissociável das tecnologias de processamento de polímeros. No entanto, pela sua importância, este tópico seria por si só merecedor de uma outra revisão bibliográfica.

\section{AGRADECIMENTOS}

A. C. C. Esteves agradece à Fundação para a Ciência e Tecnologia (FCT) uma bolsa de Doutoramento. Os autores agradecem o financiamento do projecto POCTI/35458/CTM/ 2000 da FCT (Portugal) apoiado pelo FEDER.

\section{REFERÊNCIAS}

1. http://messybeast.com/marten-cat.html, acessada em Maio 2004.

2. Dagani, R.; Chem. Eng. News 1999, 7, 25.

3. Mann, S.; Hannington, J. P.; Williams, R. J. P.; Nature 1986, 324, 565; Shenton, W.; Davis, S. A.; Mann, S.; Adv. Mater. 1996, 11, 449; Estroff, L. A.; Hamilton, A. D.; Chem. Mater. 2001, 13, 3227; Grassman, O.; Müller, G.; Löbman, P.; Chem. Mater. 2002, 14, 4530.

4. Mann, S.; Biomineralization: Principles, and concepts in bioinorganic materials chemistry, Oxford University Press, 2001.

5. Oriakhi, C. O.; J. Chem. Educ. 2000, 77, 1138.

6. Gangopadahyay, R.; De, A.; Chem. Mater. 2000, 12, 608.

7. Jiang, H.; Kakkar, A. K.; J. Am. Chem. Soc. 1999, 121, 3657.

8. Burke, N. A. D.; Stöver, H. D. H.; Dawson, F. P.; Chem. Mater. 2002, 14, 4752.

9. Walt, D. R.; Fleming, M. S.; Mandal, T. K.; Chem. Matter. 2001, 13, 2210; Sertchook, H.; Avnir, D.; Chem. Mater. 2003, 15, 1690; Seymour, R. B.; Polimeric composites-new concepts in polymer science, VSP-Ultecht: Netherlands, 1990.

10. Gilman, J. W.; Jackson, C. L.; Morgan, A. B.; Harris, R. J.; Manias, E.; Giannelis, E. P.; Wuthenow, M.; Hilton, D.; Philips, S. H. ; Chem. Mater. 2000, 12, 1866; Hsiue, G. H; Liu, Y. L.; Liao, H. H.; J. Polym. Sci. 2001, 39, 986.

11. Ou, Y.; Yang, F.; Yu, Z. Z.; J. Polym. Sci., Part B: Polym. Phys. 1998, 36, 789.

12. Zhu, J.; Uhl, F. M.; Morgan, A. B.; Wilkie, C. A.; Chem. Mater. 2001, 13, 4649 .

13. Fogg, D. E.; Radzilowski, L. H.; Dabbousi, B. O.; Scrock, R. R.; Thomas, E. L.; Bawendi, M. G.; Macromolecules 1997, 30, 8433; Qi, L.; Cölfen, H.; Antonietti, M.; Nano Lett. 2001, 1, 61; Yang, Y.; Huang, J.; Liu, S.; Shen, J.; J. Mater. Chem.1997, 7, 131.

14. Morais, P. C.; Azevedo, R. B.; Rabelo. D.; Lima, E. C. D.; Chem. Mater. 2003, 15, 3; Ramos, C.; Milan, A.; Calbet, G.; Palacio, F.; Chem. Mater. 2000, 12, 3681 .

15. Du, H.; Cao, Y.; Bai, Y. ; Zhang, P.; Qian, X.; Wang, D.; Li, T.; Tang, X.; J. Phys. Chem. B 1998, 102, 232.

16. Weller, H.; Adv. Mater. 1993, 5, 88 .

17. Steigerwald, M. L.; Brus, L. E.; Acc. Chem. Res. 1990, 23, 183.

18. Joly, C.; Smaihi, M.; Porcar, L.; Noble, R. D.; Chem. Mater. 1999, 11, 2331.

19. Ramos, J.; Palacio, F.; Polymer 2000, 41, 8461.

20. Chem. Mater. 2001, 13 (Edição especial: nanocompósitos), 10.

21. Ishizu, K.; Tsubaki, K.; Mori, A.; Uchida, S.; Prog. Polym. Sci. 2003, 28, 27.

22. Nowicki, W.; Macromolecules 2002, 35, 1424.

23. Ginzburg, V. V.; Balazs, A. C.; Polymer 2002, 43, 461.

24. Trindade, T.; O'Brien, P.; Pickett, N.; Chem. Mater. 2001, 13, 3843.

25. Meulenkamp, E. A.; J. Phys. Chem. B 1998, 102, 5566.

26. Trindade, T.; Esteves, A. C. C.; Curr. Opin. Solid State Mater. Chem. 2002, $6,347$.

27. Steigerwald, M. L. ; Brus, L. E.; Acc. Chem. Res. 1990, 23, 183; Weller H; Adv. Mater. 1993, 5, 88; Rajeshwar, K.; Tacconi, N. R.; Chenthamarakshan, C. R.; Chem. Mater. 2001, 13, 2765.

28. Trindade, T.; Neves, M. C.; Barros, A. M. V.; Scr. Mater. 2000, 43, 567.

29. Esteves, A. C. C.; Monteiro, O. C.; Barros-Timmons, A. M. V.; Boemare, C.; Soares, M. J.; Monteiro, T.; Trindade, T.; J. Nanosci. Nanotechnol. 2002, 2, 177 .

30. Bershtein, V. A.; Egorova, L. M.; Yakushev, P. N.; Pissis, P.; Sysel, P.; Brozova, L.; J. Polym. Sci. 2002, 40, 1056.

31. Fornes, T. D.; Paul, D. R.; Polymer 2003, 44, 3945.

32. Esteves, A. C. C.; Barros-Timmons, A. M. V.; Trindade, T.; Martins, J. A.; Cruz-Pinto, J.; Zhang, W.; Composites part B: engineering, no prelo.

33. Neves, M. C. ; Tese de Mestrado em Ciência e Engenharia de Materiais, Universidade de Aveiro, Portugal, 2002.

34. Wu, Q.; Liu, X.; Berglund, A. L.; Polymer 2002, 43, 2445.

35. Zanetti, M.; Camino, G.; Thomann, R.; Mülhaupt, R.; Polymer 2001, 42, 4501.

36. Liu, H.; Kim, D. W.; Blumstein, A.; Kumar, J.; Tripathy, S. K.; Chem. Mater. 2001, 13, 2756. 
37. Philipse, A. P.; Vrij, A. ; J. Colloid Interface Sci. 1989, 128, 121.

38. Bourgeat-Lami, E.; J. Nanosci. Nanotechnol. 2002, 2, 1.

39. Nassar, E. J.; Messaddeq, Y.; Ribeiro, J. L.; Quim. Nova 2002, 25, 27.

40. Foshiera, J. L.; Pizzolato, T. M.; Benvenutti, E. V.; J. Braz. Chem. Soc. 2001, 12, 159.

41. Mulvaney, P.; Liz-Marzán, L. M.; Giersig, M.; Ung, T.; J. Mater. Chem. 2000, 10, 1259; Kobayashi, Y.; Correa-Duarte, M. A.; Liz-Marzán, L. M; Langmuir 2001, 17, 6375; Salgueiriño, V. S.; Caruso, F.; Liz-Marzán, M.; J. Phys. Chem. B 2003, 107, 10990.

42. Kobayashi, Y.; Horie, M.; Konno, M.; González, B. R.; Liz-Marzán, L. M; J. Phys. Chem. B 2003, 107, 7420.

43. Piirma, I.; Polymeric Surfactants, Marcel Dekker, Inc, 1992, cap. I e IV.

44. Rajagopalan, R.; Hiemenz, P. C.; Principles of colloid and surface chemistry, $3^{\mathrm{a}}$ ed., Marcel Dekker, Inc., 1997, cap. XIII.

45. Kickelbick, G.; Prog. Polym. Sci. 2003, 28, 83.

46. Chang, T. C.; Wang, Y. T; Hong, Y. S.; Chen, H. B.; Yang, J. C; Polym. Degrad. Stab. 2000, 69, 317

47. Schottner, G.; Chem. Mater. 2001, 13, 3422.

48. Tien, Y. I.; Wei, K. H.; Polymer 2001, 42, 3213.

49. Suh, D. J.; Lim, Y. T.; Park, O. O.; Polymer 2000, 41, 8557

50. Rong, M. Z.; Zhang, M. Q.; Zheng, Y. X.; Zeng, H. M.; Friedrich, K.; Polymer 2001, 42, 3301.

51. Vaia, R. A.; Ishii, H.; Giannelis, E. P.; Chem. Mater. 1993, 5, 1694; Krishnamoorti, R.; Vaia, R. A.; Giannelis, E. P.; Chem. Mater. 1996, 8, 1728 .

52. Lemmon, J.; Lerner, M.; Chem. Mater. 1994, 6, 207.

53. Oriakhi, C. O.; Lerner, M. M.; Chem. Mater. 1996, 8, 2016

54. Sandi, G.; Joachin, H.; Kizilel, R.; Seifert, S.; Carrado, A.; Chem. Mater. 2003, 4, 838 .

55. Frisch, H. L.; Mark, J. E.; Chem. Mater. 1996, 8, 1735

56. Wilkes, G. L.; Wen, J.; Chem. Mater. 1996, 8, 1667.

57. Chen, Y.; Iroh, O.; Chem. Mater. 1999, 11, 1218.

58. Shao, P. L.; Mauritz, K. A.; Moore, R. B.; Chem. Mater. 1995, 7, 192.

59. Messersmith, P.; Stupp, S.; Chem. Mater. 1995, 7, 454.

60. Liu, J.; Gao, Y.; Wang, F.; Wu, M.; J. Appl. Polym. Sci. 2000, 75, 384.

61. Hu, Y.; Wang, S.; Ling, Z.; Zhuang, Y.; Chen, Z.; Fan, W.; Macromol. Mater. Eng. 2003, 288, 272

62. Liu, C. H.; Tsai, C. J.; Chem. Mater. 2003, 15, 320.

63. Paoli, M. A.; Alves, O. L.; Zarbin, A. J. G.; Neves, S.; Quim. Nova 2000, 23, 204; Beleze, F. A.; Zarbin, A. J. G.; J. Braz. Chem. Soc. 2001, 12, 542.

64. Percy, M. J.; Barthet, C.; Lobb, J. C.; Khan, M. A.; Lascelles, S. F.; Yamvkaki, M.; Armes, S. P.; Langmuir 2000, 16, 6913.

65. Sondi, I. ; Fedynyshyn, T. H.; Sinta, R.; Matijević, E.; Langmuir 2000, 16, 9031.

66. Matyjaszewski, K.; Pyun, J.; Chem. Mater. 2001, 13, 3436.

67. Painter, P. C.; Coleman, M. M.; Fundamentals of polymer science, $2^{\mathrm{a}}$ ed., Techonomic, 1997.

68. Bourgeat-Lami, E.; Lang, J.; J. Colloid Interface Sci. 1999, 210, 281; Bourgeat-Lami, E.; Lang, J.; J. Colloid Interface Sci. 1998, 197, 293.

69. Barthet, C.; Hickey, A. J.; Cairns, D. B.; Armes, S. P.; Adv. Mater. 1999, $11,408$.

70. Choi, Y. S.; Choi, M. H.; Wang, K. H.; Kim, S. O.; Kim, Y. K.; Chung, I. J.; Macromolecules 2001, 34, 8978.

71. Esteves, A. C.; Tese de Mestrado em Ciência e Engenharia de Materiais, Universidade de Aveiro, Portugal, 2002.

72. Xavier-Luna, J. L.; Bourgeat-Lami, E.; Guyot, A.; J. Colloid Interface Sci. 2002, 250, 82

73. Avella, M.; Errico, M. E.; Martuscelli, E.; Nano Lett. 2001, 1, 4.

\section{ABREVIATURAS}

AIBN

AIBA

AMPS

APMS

CPTMS

DBS-Na

DCPO

DMF

FTIR

GPTMS

MMA

MMT

MPTMS

PDMS

PE

PEO

PMMA

PPTMS

PS

PU

PVA

PVAc

PVI

PVP

SPTMS

TEM

TEOS

$\mathrm{T}_{\mathrm{g}}$

TGA

TMOS
Azobis-isobutironitrilo

2,2'-isobutiroamidina

Ácido 2-acrilamido-2-metil-1-propenosulfónico

Amino-propil-metoxi-silano

Cianato-propil-trimetoxi-silano

Dodecilbezenosulfonato de sódio

Peróxido de dicumilo

Dimetilformamida

Espectroscopia de Infravermelho com transformadas de Fourier

3-glicidiloxi-propil-trimetoxi-silano

Metacrilato de metilo

Montmorilonite

3-metacriloxi-propil-trimetoxi-silano

Poli(dimetilsiloxano)

Poli(éster)

Poli(óxido de etileno)

Poli(metacrilato de metilo)

Fenil-propil-trimetoxi-silano

Poli(estireno)

Poli(uretano)

Álcool polivinílico

Poli(acetato de vinilo)

Poli(vinil imidazole)

Poli(n-vinil pirrolidona)

Sulfo-propil-trimetoxi-silano

Microscopia electrónica de transmissão

Tetraetoxi-silano

Temperatura de transição vítrea

Análise termogravimétrica

Tetrametoxi-silano 